\title{
Applications of PZT Dielectric Ceramics in High-Energy Storage Systems
}

\author{
Silva Neto, L.P., a , Rossi, J.O., b and Silva Jr., A. R. ${ }^{1, c}$ \\ ${ }^{1}$ National Institute for Space Research, Associated Plasma Laboratory \\ P.O. Box 515, 12245-970, S.J. Campos, SP, Brazil \\ asilvaneto007@yahoo.com.br, brossi931@hotmail.com, 'ataidejr2003@yahoo.com.br
}

Keywords: PZT, ceramics, BST, capacitor, energy storage, nonlinear dielectrics

\begin{abstract}
The barium and strontium titanate (BST) ceramics have been used with great success as excellent dielectrics in the construction of high voltage (HV) commercial ceramic capacitors with reduced dimensions because of their high dielectric constant. However, the main point of this paper is to investigate other type of ceramic known as PZT (Lead Zirconate Titanate) normally used as piezoelectric sensors in industrial applications. The idea herein is to use the PZT ceramics as HV dielectrics for applications in high-energy storage systems by de-poling their piezoelectric properties in order to avoid dielectric damage and losses at high frequencies. For this, de-poled PZT-4 ceramic samples $(30 \mathrm{~mm} \times 2 \mathrm{~mm})$ were submitted to HV tests, in which their dielectric breakdown strength and dielectric constant variation with the applied voltage were assessed. These results obtained confirmed the use of PZT in applications that require reasonable dielectric constant stability $(<15 \%)$ with voltage and HV dielectric breakdown $(40 \mathrm{kV} / \mathrm{cm})$ for compact high-energy storage devices.
\end{abstract}

\section{Introduction}

Nowadays research involving solid dielectrics that have high dielectric constant and high voltage breakdown strength are of the particular interest in pulsed power applications, especially in the construction of compact Blumlein lines of planar geometry with parallel plates [1].

In the search of the new dielectrics for pulsed power applications, PZT ceramic composites can be good alternative, because of their high dielectric constant (in the range of 300-3000). PZTs are ceramic composites basically made of Lead $(\mathrm{Pb})$ and oxide of Zirconium $(\mathrm{Zr})$ and Titanium (Ti) normally used in industrial application as sensors, transducers, dynamic force/pressure meters and explosive detonators in ferroelectric generators, among others. In their ferroelectric phase the piezoelectric effect is observed, in which a differential voltage is produced between their electrodes when compressed or their physical dimensions are changed in the presence of an external electric field. Also they are pyroelectric materials since a differential voltage is generated when the ceramic is heated. As PZTs have crystal structure similar to barium titanate ceramics (Perovskite) they lose their piezoelectric properties when heated above their Curie temperature (thermal de-poling). Therefore, for use as dielectrics in HV pulsed power, PZT ceramics must be de-poled to remove their piezoelectric properties, avoiding in this way dielectric losses and damages caused by mechanic deformation on pulsed conditions [2].

As HV operation in pulsed power is involved, certainly dielectric voltage breakdown (BD) is always an important factor to be considered. Material electrical BD can be caused by several reasons such as degradation of the insulation material due to aging from the environment, the distance of a physical gap in relation to the insulation material, dielectric thickness, AC or DC voltage application, and in the case of pulses: amplitude, duration and number of shots. Therefore, the BD characteristic of PZT materials needs to be understood for applications in pulsed power, since it is different for every material [1].

Other important characteristic to be considered for dielectrics when used in high-energy storage systems is their linearity since, for example, in polar materials as ceramics the dielectric constant varies with the applied voltage, temperature, stress, etc. Materials, which are more linear, have 
dielectric constant that presents a weak dependence on the applied voltage and temperature, mainly, being very useful for maximizing the energy stored in the dielectrics. In view of that, this parameter is determined by measuring the capacitance of a dielectric PZT ceramic sample as function of the applied voltage $(\mathrm{C} \times \mathrm{V})$ and temperature $(\mathrm{C} \times \mathrm{T})$. As the sample dimensions are known the corresponding dielectric constant is calculated.

To characterize both dielectric properties de-poled PZT-4 ceramic samples are submitted first to $\mathrm{C} \times \mathrm{T}$ and $\mathrm{C} \times \mathrm{V}$ measurements followed by the HV BD tests on single shot condition to assess their use in pulsed power systems. Next section describes the experimental set-up for such measurements and results and discussion section gives the main results obtained so far. For comparison, the results are compared respectively to those obtained from BST dielectrics of commercial ceramic capacitors.

Finally, it is important to emphasize that the assessment of de-poled PZTs for use in high-energy storage systems as high voltage (HV) capacitors is the main objective of this paper since it has been recently noted a paucity of this research.

\section{Experimental Set-up}

The first measurement $\mathrm{C} \times \mathrm{T}$ was made in the range of $20-200{ }^{\circ} \mathrm{C}$ by placing the devices under test inside an oven with a controlled temperature ramp of $0.05{ }^{\circ} \mathrm{C} / \mathrm{s}$. A capacitance meter connected to the electrodes of the PZT or capacitor and a thermocouple attached to the device bulk were used to give respectively the values of $\mathrm{C}$ and $\mathrm{T}$ during a run of the ramp (until the maximum temperature set at $200{ }^{\circ} \mathrm{C}$ ). The respective relative dielectric constant $\mathrm{k}$ of the dielectric under test is obtained through:

$$
k=\frac{c d}{\varepsilon_{0} A}
$$

where $\mathrm{C}$ is the measured capacitance, $\mathrm{d}$ is the capacitor dielectric thickness, $\mathrm{A}$ is the capacitor dielectric area and $\varepsilon_{0}$ is the free-space vacuum permittivity.

For the $\mathrm{C} \times \mathrm{V}$ measurements a HV DC power supply was used for biasing the device under test (DUT) and four HV blocking capacitors $(40 \mu \mathrm{F})$ in series for isolating the meter input from the DC HV voltage bias (see Fig. 1). The blocking capacitance must be at least 10 times greater than the capacitance of the DUT to not cause much interference on the measurement. As the signal amplitude of the capacitance meter is much smaller than the bias DC voltage, the meter reading gives strictly a measurement of the differential capacitance for the capacitor under test $[2,3]$.

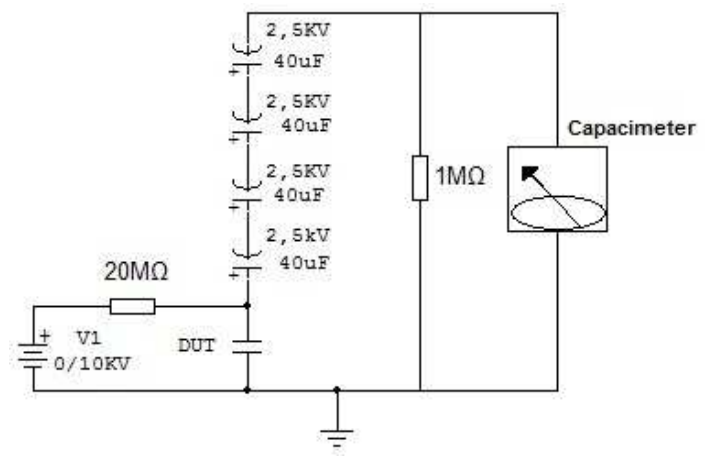

Fig. 1. Circuit used for the $\mathrm{C} \times \mathrm{V}$ measurement with $\mathrm{DC}$ bias.

For the HV BD tests, an LC oscillatory half sine wave circuit [2, 3] was used as showed in Fig. 2 (a) \& (b). This circuit is composed of two parts. The first part (a) consist of a DC power supply responsible for charging capacitor bank of $1.5 \mu \mathrm{F} / 800 \mathrm{~V}$ formed by capacitors $\mathrm{C} 3, \mathrm{C} 4, \mathrm{C} 5$ and $\mathrm{C} 6$ with same specification of $1.5 \mu \mathrm{F} / 400 \mathrm{~V}$. This capacitor bank is discharged via switch $\mathrm{S} 3$ through the primaries of two car ignition coils (model Bosch KW 12V) connected in parallel as shown in the 
second part (b) of the circuit. In order to generate a HV differential pulse on the sample to be tested, the secondary windings of both coils are biased positively and negatively. As described in part (b) of the circuit, reversed diodes connected across both coils are used to suppress the negative voltage excursion on the capacitor bank to limit input current inrush drained from the DC power supply. With full DC charging voltage a differential pulse peak of about $60 \mathrm{kV}$ is applied across the pulser positive $\&$ negative terminals. The pulse duration is about $100 \mu$ s and normally the sample breakdown near the pulse peak.

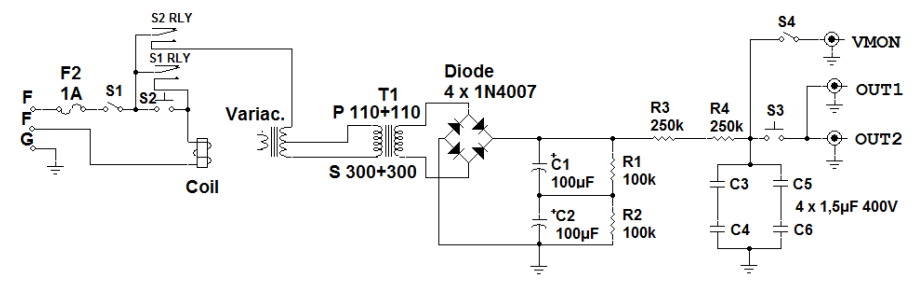

(a)

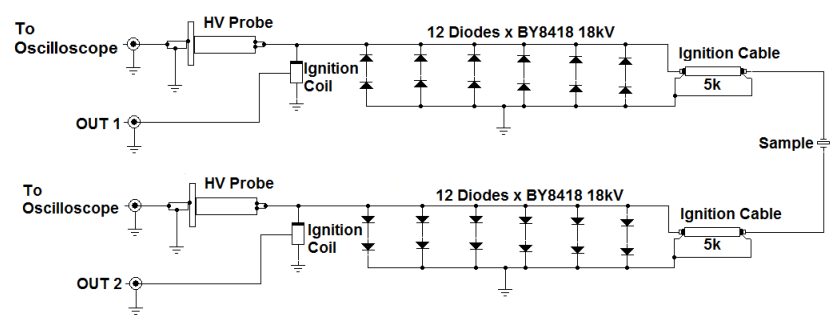

(b)

Fig. 2. Primary circuit for producing an input impulse (a), and the associated HV output circuit, showing the ignition coils (b).

\section{Results and Discussion}

The PZT-4 samples with $30 \mathrm{~mm}$ diameter and $2 \mathrm{~mm}$ thickness were acquired from a Brazilian company (ATCP) and the PZT sample de-poling thermal process has been made at factory. The samples have both (bottom and top) surfaces covered with metallic silver coating electrodes, which gives capacitances in the range of $\mathrm{nF}$. Three samples were tested, denominated PZT-D1, PZT-D2 and PZT-D3. First in Fig. 3 it is shown the $\mathrm{C} \times \mathrm{T}$ test for PZT-D3, where it can be seen clearly a nonlinear behavior with temperature since PZTs are ferroelectric materials and present an elevated Curier temperature $\left(\mathrm{T}_{\mathrm{c}}\right)$, on the order of $325^{\circ} \mathrm{C}$ in this case. As expected below $\mathrm{T}_{\mathrm{c}}$ (from room temperature up to $200{ }^{\circ} \mathrm{C}$ as shown in Fig. 3) there is an increase in the dielectric constant in blue (or capacitance in black) from 1000 up to 3250 approximately. On the other hand, compare this result with that shown in Fig. 4 for a commercial ceramic capacitor of $0.47 \mathrm{nF} / 3 \mathrm{kV}$, whose dielectric is a composite of strontium and barium titanates $\left(\mathrm{Ba}_{1-\mathrm{x}} \mathrm{Sr}_{\mathrm{x}} \mathrm{TiO}_{3}\right.$, or BST), probably with a $\mathrm{Sr} / \mathrm{Ba}$ ratio $\mathrm{x}>0.35$ to make the Curie temperature $\left(\mathrm{T}_{\mathrm{c}}\right)$ of these materials slightly lower than ambient temperature. Consequently, this ensures that the BST dielectric is in the paraelectric phase and the dielectric constant of the capacitor decreases as expected from 3000 to 750 approximately. Anyway, in both cases a nonlinear behavior is observed, but with increasing $\mathrm{C}$ (or $\mathrm{k}$ ) for PZTs at higher $\mathrm{T}$ and the opposite, i.e. decreasing $\mathrm{C}$ (or $\mathrm{k}$ ) with $\mathrm{T}$ increase for ceramic commercial capacitors. 


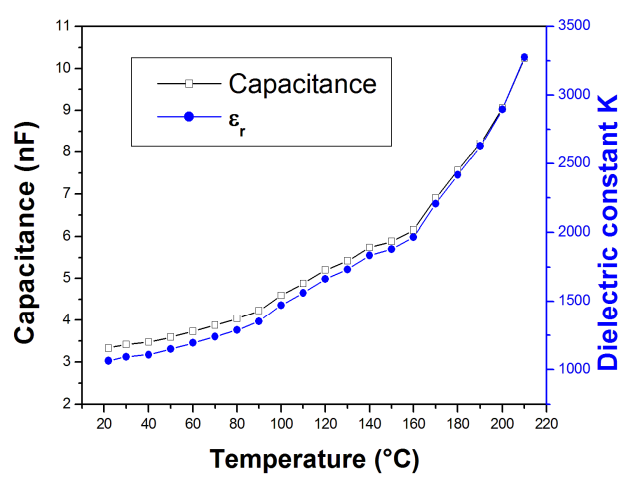

Fig. 3. $\mathrm{C} \times \mathrm{T}$ test for sample PZT-D3.

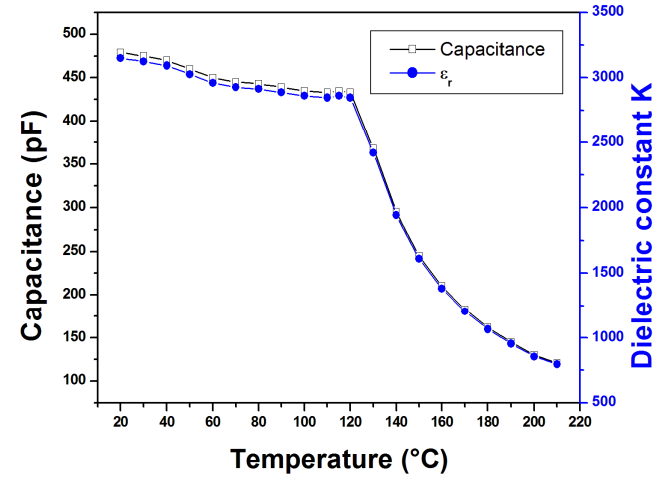

Fig. 4. $\mathrm{C} \times \mathrm{T}$ test for $0.47 \mathrm{nF}$ capacitor.

For the $\mathrm{C} \times \mathrm{V}$ tests, a tiny surface area near the border of the sample was removed and it was immersed in oil at room temperature to avoid surface breakdown. Figs. $5 \& 6$ show respectively the $\mathrm{C} \times \mathrm{V}$ tests obtained for the first PZT sample PZT-D1 and the $0.47 \mathrm{nF}$ capacitor. For the de-poled PZT dielectric tested its capacitance as a function of the applied voltage is more linear, showing a variation of only $17 \%$ (see blue line in Fig. 5) compared to the BST capacitor dielectric with $50 \%$ capacitance variation as shown in Fig. 6. A similar behavior is found for other PZT samples tested (PZT-D2 \& PZT-D3), but not presented herein due to text limit restrictions. For PZT \& capacitor, note that the dielectric constant decrease follows the capacitance variation as shown in Figs. 5 \& 6 . In both cases, dielectric constant decrease can be explained by the alignment saturation of the electric dipoles as the electric field is increased. This capacitance dependence on applied voltage is an important characteristic to be considered in the design of storing systems as the energy stored in the dielectrics $\left(\mathrm{C} \times \mathrm{V}^{2} / 2\right)$ in practice does not correspond to the ideal case. Figs. $7 \& 8$ illustrate respectively the stored energy considering the unbiased capacitance (ideal) and its variation (experimental) for PZTs (PZT-D1 \& PZT-D2) and for several ceramic capacitors $(4.7 \mathrm{nF} / 1 \mathrm{kV}, 1$ $\mathrm{nF} / 2 \mathrm{kV} \& 0.47 \mathrm{nF} / 3 \mathrm{kV}$ ). Observe in Fig. 7 that the stored energy differences between the ideal and experimental cases are very small because the PZTs are more linear with V when compared to the ceramic capacitors of Fig. 8.

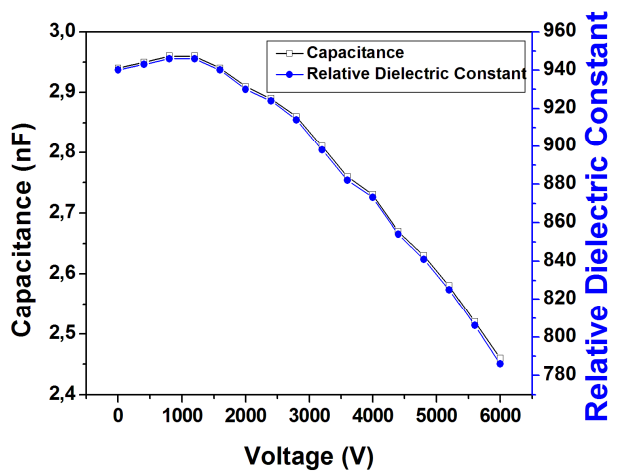

Fig. 5. $\mathrm{C} \times \mathrm{V}$ test for sample PZT-D1.

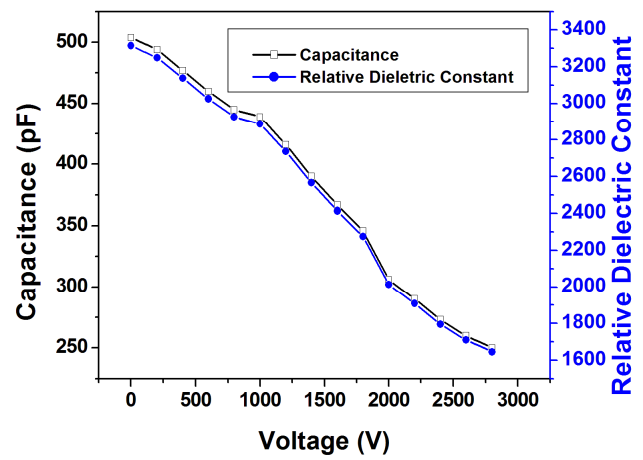

Fig. 6. $\mathrm{C} \times \mathrm{V}$ test for $0.47 \mathrm{nF}$ capacitor. 


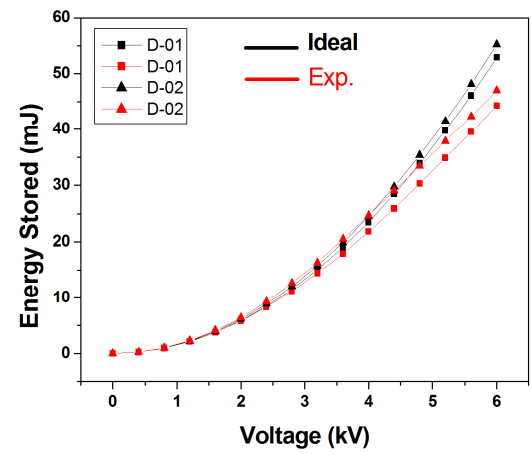

Fig. 7. Energy stored in PZT samples.

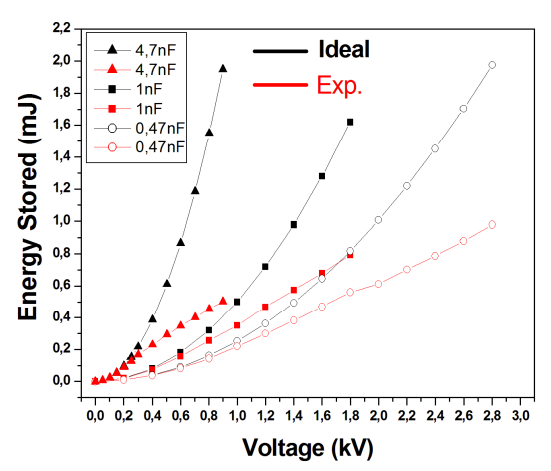

Fig. 8. Energy stored in ceramic capacitors.

For the BD tests, the capacitor terminals were directly connected to the pulser output while a second sample holder with smaller electrode area $(<5 \mathrm{~mm}$ in diameter) was built for PZT pulser connection to provide lower sample capacitances $(\leq 1 \mathrm{nF})$. For this, the PZT ceramic silver coatings were completely removed by polishing both surface sides with thin sand paper. A lower capacitance on the HV BD test is desired because causes much less attenuation on the primary voltage, allowing higher output pulse amplitudes during BD. Other point to be noted is that the devices are damaged during BD tests because of the bulk rupture. Figs. $9 \& 10$ show respectively the BD tests on single shot obtained for the sample PZT-D1 and the ceramic capacitor of $0.47 \mathrm{nF} / 3 \mathrm{kV}$. From Fig. 9, one obtains a peak-to-peak BD voltage of approximately $9.5 \mathrm{kV}$ for PZT-D1, which gives dielectric strength of $4.75 \mathrm{kV} / \mathrm{mm}$ with a PZT dielectric width of $2 \mathrm{~mm}$. Similarly from Fig. 10, a dielectric strength on the order of $8.5 \mathrm{kV} / \mathrm{mm}$ is obtained for the $4.7 \mathrm{nF}$ capacitor with a BD voltage of about $10 \mathrm{kV}$ and dielectric thickness of $1.18 \mathrm{~mm}$. Comparing with BST capacitors, these results show that PZTs have approximately half the pulsed BD strength of ceramic capacitors.

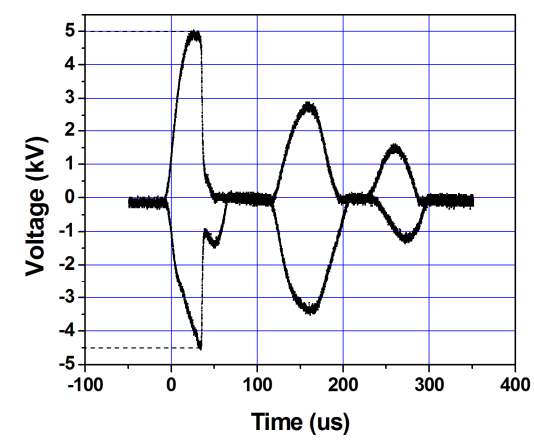

Fig.9. BD voltage test for PZT-D1.

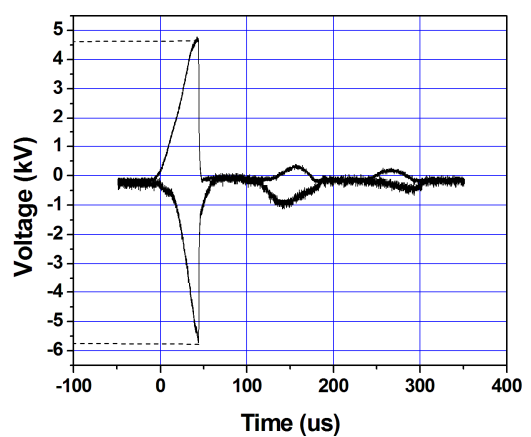

Fig.10. BD voltage test for $0.47 \mathrm{nF}$ capacitor.

\section{Conclusions}

De-poled PZT samples are much more linear with the applied voltage than BST capacitors and, therefore, they are suitable for linear applications up to $4 \mathrm{kV}$ (i.e. electric field of $2.0 \mathrm{kV} / \mathrm{mm}$ ) to avoid any residual spontaneous polarization in dielectric. It was demonstrated that the variation of the final energy stored in the dielectric is very small compared with the ideal case, which makes PZTs may be useful flat pulse generation using pulse forming lines and/or networks. However, any temperature increase in PZTs is not allowed for linear applications as they are very dependent on T and an increase in temperature can reduce the BD voltage. Also the breakdown tests show that PZTs can also be applied in pulsed linear applications until to amplitudes of about $9 \mathrm{kV}$ on single shot condition or 1.5 times their DC dielectric strength $(6 \mathrm{kV})$, considering the samples tested here. If many shots are required the $\mathrm{BD}$ voltage must be reduced to half the $\mathrm{BD}$ voltage measured to produce a dielectric mean life on the order 1000 shots, at least. Finally, planar linear transmission lines constructed from PZT ceramic dielectrics could be used for load matching and power transference, operating at half the pulsed PZT BD voltage (of $4.5 \mathrm{kV}$ approximately in our case). 


\section{Acknowledgments}

The authors acknowledge their sponsors USAF SOARD (contract number no.FA9550-10-1-0013) and Brazilian Research Council (CNPq- DF) for their support.

\section{References}

[1] P. Castro, C.J. Buchenauer, J. Gaudet and E. Schamiloglu, in: 15th IEEE Pulsed Power Conference. Monterey, June 2005. Proceeding...Monterey (2005), p. 978. (USA)

[2] J.O. Rossi, L.P. Silva Neto and A.R Silva Jr.: in $18^{\text {th }}$ IEEE International Pulsed Power Conference. Chicago, June 2011. Proceeding...Chicago (2011). (USA) to be published

[3] L.P. Silva Neto, J.O. Rossi, and A.R. Silva Jr., in: XI Brazilian Power Electronics Conference. Natal, September 2011. Proceeding...(Natal), iREP-412. (RN). 
Advanced Powder Technology VIII

10.4028/www.scientific.net/MSF.727-728

Applications of PZT Dielectric Ceramics in High-Energy Storage Systems

10.4028/www.scientific.net/MSF.727-728.505 